\title{
Wie Kranke, deren eines Auge am Staar operirt ist, selien, und über die Frage, ob einseitige Cataract- operation und einseitige Pupillenbildung, bei gesundem zweiten Auge, zweclidienlich sei?
}

Von

\author{
Dr. A. v. Gräfe.
}

Es ist gegen die Operation des grauen Staars auf einem Auge, wenn das andere Auge gesund ist, von vielen Seiten her der Einwurf erhoben worden, dass selbst das beste Gelingen keine Vortheile für den Kranken bringe, weil ja der Unterschied der Brechkraft nach wieder erlangtem Sehvermögen auf dem kranken Auge an ,jedem gemeinschaftlichen Sehakt hindere. Ja man hat sogar wesentliche Nachtheile und Störungen eines solchen Verfahrens gefürchtet, sofern die Zerstreuungskreise des hyperpresbyopischen (operirten) Auges die deutlichen Bilder des anderen bedecken und verwischen sollten, wodurch die Kranken genöthigt wären, entweder das eine Auge willkührlich zu schliessen, oder durch Muskelcontraktion in eine schielende Lage zu bringen.

Wenn ich num in einer sehr grossen Reihe von Fällen (uiber 50 ), wo ich die Cataraktoperation auf 
einem Auge vollführte, während das andere gesund war, im Wesentlichen nichts von diesen Uebelständen verspürte, so lag mir gewissermaassen die Pflicht ob, die Rolle genau zu bestimmen, welche das operirte Auge beim gemeinschaftlichen Sehakt übernimmt. Es zeigte sich hierbei, dass die Verhältnisse ausserordentlich verschieden sind.

1) In einer grossen Zahl von Fällen wird bei gemeinschaftlichem Oeffuen beider Augen das Bild, welches vom operirten Auge herrührt, aus der Wahrnehmung ausgeschlossen, wie dies ja auch sonst häufig bei verschiedener Brechkraft beider Augen geschieht. Der Beweis hierfür wird dadurch geliefert, dass keine absolut scharfe Fixation bei den accommodativen und associirten Bewegungen mit dem operirten Auge eingeleitet wird. Eine absolut s charfe Fixation für die verschiedenen Verhältnisse des Sehakts ist ja an eine Wahrnehmung des Netzhaut-Eindruckes gebunden, da das Gefühl der Muskelcontraktion nur in approximativer Weise die Einstellung reguliren kann. Wir finden deshalb, dass alle Individuen, die nur ein Auge zum Sehakt benutzen, das zweite Auge nur ung e fähr*) und für gewisse Lagen des Gesichtsobjekts einstellen, während dasselbe für andere, den Muskeln weniger geläufige Lagen, z. B. bei forcirter Annäherung des Objekts, oder beim Blick nach oben, von der ihm zukommenden Lage sichtbar abweicht. Noch genauer lässt sich diese Exclusion des einen Auges beim Sehakt daran erkennen, dass wir

*) Der Nachweis liegt in der Verrückung, welche in diesem zweiten Auge eintritt, wennn das zum Sehakt benutzte Auge geschlossen wird. Diese Verrïckung ist oft ungemein klein, nur bei grösster Aufmerksamkeit oder teleskopischer Beobachtung zu konstatiren und deren Richtung nicht einmal immer zu bestimmen, weil sich kurze gewissermassen suchende Bewegungen in entgegengesetzter Richtung succediren, ehe es zur Fixation kommt. 
durch Vorhalten von Prismen weder Doppelbilder noch eine Ablenkung der Sehaxe erhalten. Eins von beiden müsste aber Statt finden, wenn das Netzhautbild dieses einen Auges mit dem des andern in einen gemeinschaftlichen Eindruck verschmolzen war; es müsste entweder unter Beibehaltung der früheren Lage ein excentrisches Bild entstehen (und sich dann als Doppelbild oder Nebenbild aus dem gemeinschaftlichen Eindrucke sondern), oder es müsste durch eine willkührliche Veränderung in der Augenstellung der brechenden Einwirkung des Prismas so entgegengetreten werden, dass der gebrochene Lichtstrahl in Richtung der Sehaxe, und demnach auf die macula lutea fällt. Tritt keins von beiden ein, so haben wir Grund zu schliessen, dass eine aktive Betheiligung des zweiten Auges für die qualitative Wahrnehmung nicht vorhanden war.

Die ausbleibende Wahrnehmung der Bilder, welche von dem operirten Auge stammen, wird einige Stumpfheit dieses Auges zur Folge haben, und es werden sich die Verhältnisse ganz eben so gestalten, wie bei so vielen Individuen, die wegen Unterschiedes in der Brechkraft, oder wegen Unregelmässigkeit in den brechenden Medien, oder wegen einseitiger Schwachsichtigkeit nur das eine Auge zur Wahrnehmung benutzen. Das excludirte Auge verfällt in eine gewisse Stumptheit, als Folge des Nichtgebrauchs, welche wir einer methodischen Separatübung oder auch spontan dann weichen sehen, wenn das früher gesunde Auge durch ein accidentelles Uebel erblindet.

Diese Form von einseitiger Schwachsichtigkeit ist zwar wohl einem jeden Augenarzt bekannt, dürtte aber doch noch einmal hervorgehoben werden, weil wir in der gewöhnlichen Praxis nur zu häufig in Betreff derselben therapeutische und prognostische Irrschlüsse hören. So kommt es, um mich an eins der häufigsten Beispiele zu halten, vor, dass Individuen auf dem einen Auge mit 
einer leichten Hornhauttrübung, aber unverhältnissmässig starker Schwachsichtigkeit behaftet sind. Lässt man dieselben das kranke Auge entweder für sich oder mit geeigneten Convexglüsem üben, so stellt sich die Sehkraft bis auf einen gewissen Punkt wieder her, natürlich ohne dass die Hornhauttrïbung sich im Mindesten geändert hat. Werden etwa irgend welche Arzneimittel angewandt, und gleichzeitig solche Uebungen gemacht, so wird auch die erlangte Besserung nicht selten irriger Weise auf die Lichtung der Hornhaut geschoben. Das Verhältuiss ist hier so, dass die Hornhauttrübung, sei es wegen der dadurch bedingten Schwachsichtigkeit oder wegen gleichzeitigen Unterschiedes in der Brechkraft (z. B. durch vermehrte Wölbung der Hornhaut), oder wegen der ihr zukommenden Lichtzerstreuung, den Grund abgab für die Exklusion des Auges von dem gemeinschaftlichen Sehakt, und dass diese Exklusion ihrerseits die zunehmende Sehschwäche hervorrief. Es wird demnach auch die Uebung des betroffenen Auges die Sehkraft soweit restituiren können, als es die Hornhauttrübung zulässt. Dasselbe gilt wie von Hornhauttrübungen so auch von Linsentrübungen, Amblyopieen der verschiedensten Art, Refraktionsunterschieden, kurz allen Verhältnissen, welche zur Exclusion des einen Auges führen können. Es sei übrigens in Betreff dieser aus Nichtgebrauch des einen Auges resultirenden Sichwachsichtigkeit bemerkt, dass sie für sich, abgesehen von den ihr zu Grunde liegenden materiellen Störungen, nie einen sehr hohen Grad zu erreichen pflegt, und dass die Leitung auf der Netzhaut dabei vollkommen intakt bleibt. Niemals dïren wir amaurotische Amblyopieen, bei denen nur noch gröbste Objecte, und mit Convexgläsern nicht mehr Lettern erkannt werden können, lediglich auf Nichtgebrauch reduciren. Nur wemn in den ersten Lebensjahren die Exklusion eintritt, köunte von dieser Regel eine Ausnahme stattlinden*). Die Integrität in der Leitung der Netzhaut spricht sich dadurch aus:

*I Ich habe diesen Gegenstand schon früher einmal (A. f. 0 . Bd. I., Heft I., S. 329) lerührt, und auf den Unterschied aufmerksam gemacht, der zwischen der Exklusion des einen Auges. z. B. bei einseitiger Catarect, bei aufgehobenem Lichteinfall, Pupillen- 
1) dass das Gesichtsfeld an Umfang nichts verloren;

2) dass das excentrische Sehen relativ zum centralen vollkommen seine Deutlichkeit erhalten, oder eher etwas vermehrt hat;

3) dass die Störung eben nur in der Zunahme des zum Erkennen nothwendigen Gesichtswinkels besteht; dass demgemäiss das Erkennen proportional zur Vergrösserung des Gesichtswinkels steigt. Es basirt hierauf der allbekannte giinstige Einfluss der Convexgläser bei der ans Nichtgebrauch hervorgegangenen Amblyopie, wobei wir natürlich von Complikationen mit Accommodationskrankheiten abstrahiren müssen.

Die Zeichen der erhaltenen Leitung auf der Netzhaut sind ausserordentlich wichtig für die aus Nichtgebrauch resultirende Amblyopie, denn wenn sie gleich die letztere nicht diagnostisch characterisiren, vielmehr noch anderen Krankheitsformen zukommen, so liefern sie doch gegen manche Gruppe der Amblyopieen scharfe Unterscheidungsmerkmale.

Die Schwachsichtigkeit des schielenden Auges kann deshalb nicht allein auf Nichtgebranch beruhen, wejl häufig unmittelbar nach der Sebnendurchschneidung eine nambafte Besserung eintritt, dass aber die Exklusion einen gewissen Antheil an der Sehschwäche des schielenden Auges hat, ist audrerseits nicht zu bezweifeln, sofern wir ja auch ohne Tenotomie, und demnach ohne Beseitigung des Schielens durch die geeignete Separatübung des schielendeu Auges einige Besserung der Sehkraft erreichen.

2) Weit interessanter gestalten sich die Verhältnisse in andern Fällen nach einseitiger Cataractoperation, in welchen nämlich das betreffende Auge gleichzeitig mit dem zweiten zum Sehakt verwendet wird, ohne dass aus der enormen Verschiedenheit der Brechkraft

verschluss etc. und zwischen der Exklusion (aus der Wahrnehmungi bei zu Stande kommenden Netzhautbildern stattindet. Dass die erstere Ursache gar keine oder sehr geringe Stumpfheit erzeugt, schloss ich damals aus dem Falle einer seit 60 Jahren reifen, durch Extraction operirten Katarakt. Ich könnte diesem Falle heute noch mehrere hinzufügen von Catarakten, die seit 40 und 30 Jahren bestanden, und wo sich die Verhältnisse des Sehens ganz wie dort verhielten. 
irgend ein für den Kranken bemerkbarer Nachtheil entspringt. Ich habe dies wiederholentlich an jüngeren Individuen beobachtet, deren eines Auge an weicher Cataract erblindet und durch lineare Extraktion wiederhergestellt war. Schon bei rohen Versuchen fiel die vollkommen scharfe Fixation, bis zur grössten Annäherung des Objectes, auf. Die Betheiligung des operirten Auges beim Sehakt ergab sich auf das Evidenteste bei gründlicher Prüfung der oben angegebenen Kriterien. In allen Lagen des Gesichtsobjekts war das operirte Auge in der Fixation, denn beim Verschluss des gesunden trat nicht die geringste Verrückung in der Sehaxe ein. Jeder Rest von Zweifel, der wegen der Schwierigkeiten kleine Zuckungen wahrzunehmen, etwa noch bleiben konnte, wurde vollends durch die Anwendung prismatischer Gläser beseitigt. Hielt man ein Prisma zwischen $6^{\circ}-10^{\circ}$, mit der Basis nach aussen, vor das operirte Auge, und liess ein mehrere Fuss distantes Licht fixiren, so erschien das Licht dem Kranken einfach, aber es trat unter dem Prisma sofort ein convergirendes Schielen ein; wurde das Prisma hinweggenommen, so wich die Sehaxe in ihre frühere Lage nach aussen zurück. Wurde ein Prisma zwischen $10^{\circ}$ $-16^{0}$ gewählt, und ebenfalls mit der Basis nach aussen gehalten, so trat bei dem 范heren Abstarde des Lichtes kein willkührliches Schielen mehr ein, aber der Kranke sah gleichnamige Doppelbilder, deren Entfernung unter den gegebenen Verhältnissen der Ablenkungskraft des Prisma entsprach. Wurde das Licht unter Beibehaltung desselben Prisma bis auf $10^{\prime \prime}$ resp. $6^{\prime \prime}$ genähert, so konnten die Doppelbilder zusammen gebracht werden, wobei ein beträchtliches willkührliches Schielen nach innen sich einstellte; wurde nun das Licht wieder entfernt, so konnte das Einfachsehen bis auf einer 
nach dem Winkel des Prisma zwischen $2^{\prime \prime}$ und $10^{\prime \prime}$ variirenden Grenze erhalten werden, worauf wieder Doppelsehen entstand, und mit dem Auseinanderweichen der Bilder wich die Sebaxe aus ihrer schielenden Stellung nach aussen hin ah. Wenn dass Prisma statt mit seiner Basis nach aussen nach innen hin angelegt wurde, so entstand bei ganz schwachem Prisma $\left(4^{0}-6^{0}\right)$ ein divergirendes Schielen, bei stärkeren Prismen Doppelsehen mit gekreuzten Bildern; selbst die schwächsten Prismen, mit der Basis nach unten resp. nach oben angelegt, hatten sofort übereinanderstehende Doppelbilder zur Folge. Es blieb demnach nicht der geringste Zweifel, dass in den betreffenden Fällen das Bild des operirten Auges mit dern gesunden in eine gemeinschaftiche Wahrnehmung verschmolzen war, eine Thatsache, die bei solchem Unterschiede in der Brechkraft allerdings etwas Räthselhaftes in sich trägt. Der Zerstreuungskreis, den das operirte Auge liefert, muss ja relativ zum scharfen Sehen des gesunden Auges so diffus sein, dass wir kaum begreifen, wie ein gerneinschaftliches Sehen ohne Störung der Sehschärfe möglich ist. - Es zeigten sich in diesen Fällen auch alle Vortheile des gemeinschaftlichen Sehens, vor allen Dingen eine höchst sichere Taxation der Entfernungen und körperliches Sehen, welches verschwand, wenn das operirte Auge verdeckt wurde.

Diese Thatsache beweist aufs neue, wie sehr das Urtheil den Sehakt influenzirt, und wie es sich über Unvollkommenheiten desselben hinwegzusetzen weiss. Sie schliesst sich in dieser Weise an die Ueberdeckung des blinden Flecks, an die Verträglichkeit gegen schiefe Doppelbilder und derlei an. Etwas Aehnliches finden wir übrigens in den so häufigen Fällen von verschiedener Brechkraft beider Augen; auch hier entstehen niemals auf beiden Seiten scharfe Bilder. Selbst dann, 
wenn das Accommodationsbereich des cinen Auges mit dem des andern Auges zum Theil zusammenfällt (indem der Fernpunkt des kurzsichtigern Auges entlegener ist als der Nahepunkt des fernsichtigren Auges), waltet doch beim doppelseitigen Sehen nie ein gleicher Accommodationszustand. Es herrscht gerade zwischen den Accommodationsimpulsen cine ausserordentliche Sympathie in beiden Augen, und es kann diese selbst in der Absicht, gleich scharfe Bilder zu erbalten, nicht in der Weise gebrochen werden, dass das eine Auge für seine Nahepunktsgegend, das andere für seine Fernpunktsgegend accommodirt ist. Man kann sich auch durch Experimente überzeugen, dass der Accommodationszustand unter solchen Verhältnissen in beiden Augen stets ein verschiedener ist, und immer nur das eine Auge ein scharfes Bild bietet. Beim Verschluss dieses einen Auges tritt erst auf dem zweiten die vollkommen genaue Einrichtung des Brechzustandes ein, wic dies zum Theil das subjective Gefïhl der Kranken, zum Theil objective Erschrinungen, insonderheit eine Veränderung in der Richtung der Sehaxe auf dem verdeckten Auge, nachweist (siehe A.f. O. Bd. II., 1. S. 167). Trotz alle dem hetheiligen sich bei vielen derartigen Kranken wirklich beide Augen gemeinschaftlich am Sehakt, und die Störungen des einseitigen Zerstreuungskreises werden demnach unterdrückt, wie in dem obigen Fall*).

Dass die verschiedene Lichtstärke der Bilder den gemeinschaftichen Gebrauch nicht ausschliesst, ist eine vollkommen festgestellte Thatsache, welche jedoch nieht

*) Wir wissen sehr wohl, dass neben dieser Reihe von Fällen eine andere, sicher noch zahlreichere vorkommt, wo bei Unterschieden in der Brechkraft das eine Auge vom gemeinschaftlichen Sehakt ausgeschlossen ist, und es sind demnach die Verhältnisse eben so getheilt, wie nach einseitiger Staaroperation. 
so viel Auffallendes hat, als die oben in Rede stehende. Dagegen bietet wieder eit Analogon die bei manchen Individuen mit verschiedener Accommodation mögliche Ausgleichung der Brechkräfte durch die geeigneten Gläser. Wenn wir nämlich ein kurzsichtiges Auge durch Hinzufügung eines Concavglases in denselben Brechzustand versetzen, wic ein zweites normal brechendes Auge, so wird doch immer ein Unterschied in der Grösse des Netzhautbildes auf beiden Augen existiren. Dioptrischen Grundsätzen gemäss wird nämlich die Knotenpunktsregion auf dem mit dem Concavglas combinirten Auge der Netzhaut näher liegen, und es werden deshalb die Bilder kleiner ausfallen. Umgekehrt wird es sich verhalten, wenn ein weitsichtiges Auge durch Combination mit einem Convexglase normale Brechkraft bekommt; es werden alsdann die Knotenpunkte der Cornea mehr genähert liegen, als auf dem gesunden Auge, und demnach grüssere Bilder entstehen. Wenn trotz dieser Unterschiede, die sich hei der Berechnung allerdings als nicht sehr erheblich herausstellen, zuweilen eine Ausgleichung möglich ist, selbst bei Individuen, deren cines Auge ein Convexglas, und deren anderes ein Concavglas trägt, so spricht dies wieder für die Verträglichkeit des Sehakts mit ungenauen optischen Verhältnissen. Im Uebrigen sei hier bemerkt, dass eine solche Ausgleichungsfähigkeit nicht so in der Regel liegt, wie es manche Aerzte. und namentlich die Brillenverkäufer, denken. Man ïberzeıgt sich bei genauer Untersuchung sehr häufig davon, dass gegen den Anschein doch nur der Eindruck des einen Auges für den Sehakt benutzt wird. Für sehr viele Kranken bringt auch die verschiedene Bildgrösse solche Störung, dass man auf den Plan einer Ausgleichmg verzichten, und den einseitigen Gebrauch nebt separater Uebung des exkludirten Auges praktisch vorziehen muss. 
Kommen wir nun von diesen physiologischen Thatsachen auf die praktische Frage zurück, was von der einseitigen Cataract-Operation zu halten sei. Könnten wir nach derselben auf einen gemeinschaftlichen Gebrauch der Augen rechnen, so würde für den Kranken der grosse Vortheil einer sicheren Taxation der Entfernungen und des körperlichen Sehens resultiren, Eigenschaften, an deren Mangel sich hauptsächlich das Gebrechen der Einäugigkeit knüpft.

Meine Beobachtungen sind in der That noch nicht umfassend genug, um numerisch zu begründen, wie oft nach einseitiger Cataract-Operation ein gemeinschaftlicher Gebrauch der Augen zu Stande kommt, und unter welchen spezielleren Verhältnissen sich hierzu die grösste Aussicht eröffnet. Bei mehreren jüngern Individuen, die ich hintereinander auf einer Seite durch lineare Extraction an Cortikalstaar operirte, fand ich dieselben Verhältnisse des gemeinschaftlichen Schens. Bei ältern Patienten habe ich es erst einmal gefunden, gestehe aber auch, dass die Gelegenheit sich sehr selten darbot, da ich nicht gern eine Lappen-Extraktion, niemals aber eine Reklination auf dem einen Auge verrichte, wenn das zweite gesund ist.

Betrachten wir nun bis zu genaueren statistischen Ergebnissen vorläufig den Eintritt eines gemeinschaftlichen Sehaktes als einen glïcklichen A usn ahmefall, und fragen wir, welche Vortheile allgemeinhin für die Kranken aus der einseitigen Cataract-Operation hervorgehen. Es sind dies erstens der kosmetische Gewinn. Dieser darf uns nur zu einer vollkommen ungefährlichen Operation verleiten, z. B. zu einer linearen Extraktion bei jugendlichen Individuen, die durch ihre nachweisbare Einäugigkeit in ihrer Laufbahn nicht selten wesentlich behindert sind; so habe ich selbst bei bestehenden Amaurosen durch Netzhautablösung die 
lineare Extraktion des consekutiven Cortikalstaars verrichtet. Zweitens die Erweiterung des Gesichtsfeldes, ein sehr gewichtiger Vortheil, weil sich an ihn ein weit freieres Orientirungsvermögen und eine symmetrische Kopthaltung knüpft. Drittens, wenn auch nicht konstant, eine gewisse Zunahme in der Energie der Sehkraft, welche ich schon früher für das schielende Auge besprochen, und über welche ich bei Gelegenheit der einseitigen Pupillenbildung noch Einiges bemerken werde.

In Anbetracht dieser Vortheile würde kein Mensch sich gegen die einseitige Cataract - Operation erklären, wenn nicht auf der anderen Seite gewisse Bedenken sich erhoben hätten, welche wir ebenfalls näher ins Auge fassen müssen. Solche sind zunächst in dem unsichern Gelingen der Cataract-Operation selbst begründet. Bei jugendlichen Individuen fällt dies Bedenken, weil wir die glückliche Beseitigung der Cataract als etwas nahezu sicheres ansehen können; es gilt dies sowohl für richtig moderirte discisio per corneam, als für lineare Extralition; beide sind, unter treffenden Indikationen ausgeübt, von bösen Zufällen frei. Bei älteren Individuen aber, wo wegen Vorhandensein eines scleromatösen Linsenkerns die erwähnten Operationsweisen so gut wie ausgeschlossen sind, und es sich lediglich um Lappen-Extraction, resp. Dislokation der Linse handelt, ist das Resultat immer ein mehr oder weniger chancöses. Wir werden nach der LappenExtraktion zuweilen Vereiterung des Lappens, nach der Reklination Irido-Chorioideitis bekommen. Wenn es sich darum handelt, einem Erblindeten das Sehvermögen wieder zu geben, so fallen diese schlechten Chancen nicht als Contra-Indikationen in Betracht, weil sie durch die guten Chancen, besonders bei der Extraktion, bedeutend überwogen werden. Handelt es sich 
dagegen darum, nur das eine arblindete Auge zu restituiren, so sind die Vortheile, selbst im günstigsten Falle, dass ein gemeinschaftlicher Gebrauch der Augen eintritt, unvergleichbar klein zu denen der Wiederherstellung des Sehvermögens überhaupt, und es wird daher eine jede ungünstige Chance verhältnissmässig sehr schwer in die Waagschale fallen. Vollends zu proscribiren ist hier die Reclination, denn es ercignen sich Fälle, wo die inneren Entzïndungen, die nach Reklination entstehen, zu ciner Erkrankung des gesunden $\mathrm{Au}$ ges führen, in ähnlicher Weise wie die irido-chorioideitis nach eingedrungenen fremden Körpern zuweilen eine sympathische Affektion des zweiten Auges hervorruft. Es ist wahr, dass diese Fälle selten sind, so selten, dass erfahrene Praktiker ihr Vorkommen läugnen. Ich selbst habe deren aus Anderer Praxis vier gesehen; einmal war unmittelbar nach ausgefïhrter einseitiger Reklination Irido-Chorioideitis in beiden Augen ausgebrochen, in den anderen drei nach Wochen, resp. Monaten im deutlichen Zusammenhang mit Exacerbationen der chronischen Entzündung auf dem operirten Auge. Sollte nun selbst die Zerstörung des operirten Auges, frühzeitig verrichtet, dem Ruin des zweiten vorbeugen, in ähnlicher Weise wie es für traumatische Irido-Chorioideitis versichert wird, so ist doch das blosse Vorkommen einer sympathischen Affection auf dem zweiten Auge, mag es noch so selten sein, genügend, um den Stab über die Reklination auf' einem Auge bei gesundem zweiten zu brechen. Selbst abgesehen von einer wirklichen materiellen Erkrankung des zweiten Auges, hindert die langwierige innere Reizung des durch Reklination operirten nicht selten völlig am Gebrauch des Gesichts, und auch fïr diesen Fall hat der Kranke wesentliche Nachtheile. - Anders verhält es sich in dieser Beziehung mit der Extraktion. 
Niemals habe ich durch die Vereiterung des Lappens Zufällo: auf dem zweiten Auge entstehen sehen, und ist dies, soviel ich weiss, zur Zeit auch von keinem Beobachter anders befunden worden. Es beziehen sich demnach die üblen Chancen der Extraktion nur auf das eine operirte Auge; auch wird der ungünstigste Ausgang niemals zu langwierigen inneren Entzündungen, welche den Gebrauch des gesunden Auges autheben und geniren, führen, wie dies bei Reklination der Fall ist. Es ist demnach die einseitige Lappen-Extraktion bei gesundem zweiten Auge nur im Allgemeinen nicht räthlich, weil die Vortheile nicht in einem für die Praxis erfreulichen Verhältniss zu den üblen Chancen stehen, während die einseitige Reklination auf das Entschiedenste zu verwerfen ist. Die erstere kann sogar unter Umständen zulässig erscheinen, wenn z. B. gewisse Patienten durch die Idee, einäugig zu sein, im Falle einer doppelseitigen Erkrankung vollends zu erblinden etc., moralisch sehr deprimirt sind, den Chirurgen um die Operation inständig bitten und die etwaige Ent. stellung nach suppuration des Lappens in ihrer Vorstellung nicht höher anschlagen als die früher bestehende Blindheit u. s. w.

Die weiteren Bedenken gründen sich auf Nachtheile, welche nach glïcklicher Wiederherstellung descataractösen Auges eintreten sollen. Zunächst hat man befürchtet, dass die verschiedene Brechkraft für die Ausdauer und Beharrlichkeit des Sehakts wesentlich störend ist. Dies habe ich unter mehr als 50 einseitigen Cataract-Operationen niemals gefunden. Die Kranken konnten mindestens eben so gut und lange arbeiten, als zuvor. Zweitens hat man Blendung beim hellen Licht aut dem operirten Auge vorgeschützt; dies habe ich vielleicht in einem Viertheil der Fälle während der ersten Wochen 
oder Monates beobachtet. Fs wich aber immer von selbst, und fällt demnach nicht in Betracht. Drittens befürchtet man Schielen des operirten Auges. Ein solches im cosmetischen Sinne, d. h. eine auffallende Abweichung der Sehaxe, habe ich im Ganzen zweimal, und zwar schon in den nächsten Wochen nach der Operation, sich entwickeln sehen. Es verhält sich hiermit wie überhaupt mit dem Schielen bei verschiedener Brechkraft. Es kann Schielen daraus hervorgehen, aber es ist dies keineswegs nöthig, da sowohl ein gemeinschaftlicher Gebrauch der verschieden brechenden Augen als eine Suppression des einen Netzhautbildes bei approximativ richtiger Stellung des betreffenden Auges möglich ist. Wenn wir nun, abgesehen von der Seltenheit dieses Ausgangs, erwägen, dass ein auf verschiedener Brechkraft beruhendes Schielen trotz der fortbestehenden Ursachen doch durch die Tenotomie geheilt werden kann, ferner, dass auch ohne die CataractOperation auf einem einseitig erblindeten Auge häufig genug Schielen eintritt, so dürfte hierin keine Contraindikation für den Praktiker liegen. Viertens warnt man wegen Doppelsehens, entstehend durch eine leichte Abweichung des einen Auges auf Grund des störenden Zerstreuungskreises. Auch dies ist keinewegs häufig, ich habe es nur dreimal beobachtet. In zwei von diesen Fällen erklärte es sich durch eine schon vor der Operation bestehende Convergenz des cataractösen Auges und hörte allmählig von selbst auf, ohne dass irgend ein bedeutender Grad von $\mathrm{Ab}$ lenkung sich eingefunden hätte. Wurde ein rothes Glas vor das gesunde Auge gehalten, so konnten auch später noch Doppelbilder, ungefähr in der ursprünglichen Entfernung, gesehen werden. Es verhielt sich hier also, wie so häulig nach Strabismus-Operation, wo 
die von geringer pathologischer Convergenz herrïhrenden gleichnamigen Doppellilder bald ganz übersehen werden, wenn nämlich eine Suppression des einen Bildes eingeleitet wird. In dem dritten Falle zeigten sich grkreuzte Doppelhilder, einer leichten Divergenz des operirten Auges entsprechend. Irh bin ausser Stande zu sagen, ob diese Divergenz schon vor der Operation zugegen war oder nicht, da bei aufgehobenem Sehakt natürlich jede Beurtheilung für die genauere Stellung fehlt. Dieses Doppelsehen erwies sich sehr hartnäckig, und genirte den Kranken noch nach 8 Wochen.

Wir dürfen uns hierüber nicht wundern, wenn wir uns an das erimnern, was ich anderen Ortes über den Unterschied der gekreuzten und gleichnamigen Doppelbilder gesagt habe. Ich kann in dieser Beziehung meine trüheren Behauptungen nur durch neue Belege unterstützen. So fand ich etliche Kranke, denen ich gekreuzte Doppelbilder künstlich hervorrufen konnte, während bei gleichnamigen sofort eine Suppression eintrat. Schieloperirte, bei welchen die gemeinschaftliche Einstellung nur in einem beschränkten Terrain vor sich ging, gaben sehr häufig die bei starker Annäherung des Objekts in der Medianebene entstehenden gekreuzten Doppelbilder spontan an, während die jenseits der Einstellungsgrenze zu vermuthenden gleichnamigen Doppelbilder selbst mit gefärbten Gläsern schwer hervorzurufen waren. Auch Gesunde, welche ihr physiologisches Doppelsehen prüfen, sehen leichter die gekreuzten als die gleichnamigen Doppelbilder, und wcrden zuweilen durch die ersteren mehr verwirrt, zum Schwindel angeregt, etc.

Da sich nun boi dem Patienten mit prismatischen Gläsern nachweisen liess, dass eine Verschmelzung der beiderseitigen Eindrücke überhaupt nicht zu Stande kam, so verwandelte ich durch eine Tenotomie des externus die gekreuzten Doppelbilder in gleichnamige (natürlich mit mässigem gegenseitigen Abstande, um nicht einen sichtbaren Strabismus convergens zu veranlassen), 
worauf sich das Doppelsehen, wie in den früheren Fällen, rasch verlor*). - Fünftens könnte man behaupten, dass bei der Suppression des (physikalisch zu Stande kommenden) Netzhautbildes auf dem operirten Auge dieses in eine grössere Sehschwäche gerathe, als wenn überhaupt der Lichteinfall von demselben ausgeschlossen sei. Nach dem, was wir früher über die aus Nichtgebrauch entstehende Schschwäche gesagt, dürfen wir dies Argument nicht ganz zurückweisen, doch ist es leicht, durch die Separatübung des operirten $A u-$ ges mit Convex-Gläsern diesem Uebelstande entgegenzutreten.

Hiernach wird sich der Grundsatz ergeben: dass in Summadie Cataract-Operation auf einem $\Lambda$ uge neben wichtigen Vortheilen keine erheblichen Nachtheile bietet, und deshalb immer angezeigt ist, wenn wir auf deren Gelingen mit Sicherheit rechnen können.

Ganz anders gestaltet sich natürlich die Frage, wenn das zweite Auge nicht vollkommen gesund ist, sondern ebenfalls in vorrückender cataractöser Erkrankung steht. Alsdam werden selbst Verfahrungsweisen indicirt sein, deren Gelingen nicht durchaus sicher genannt werden kann. Offenbar müssen wir es tür eine grosse Wohlthat halten, der oft so sehr deprimirenden Zeit vorzubeugen, in der die Kranken durch Abnahme des Gesichts ihrer Selbstständigkelt mehr und mehr verlustig gehen. Es ist hierbei nur darauf zu sehen, dass die Operation in keiner Weise nachtheilig auf das noch sehende Auge influirt, und demgemäss würde ich unter diesen Verhältnissen ebenfalls nie zur Reclination rathen; dagegen ist es meine gewöhnliche Praxis, wenn es anders in den Wünschen

*) Interessant waren mir mehrere Kranke, bei denen ein vor der Operation deutlich nachweisbares divergirendes Schielen sich nach Wiederherstellung der Sehkraft allmählig verlor, indem die anfangs sichtbaren Doppelbilder durch stärkere Bethätigung des musc. rectus internus in einander getrieben wurden. 
der Kranken liegt, die Lappenextraktion auf dem einen Auge damn auszuführen, wenn der Staar auf demselben gereift, aber auf dem andern noch in der Entwicklung ist. Die Chancen tür das operirte Auge dependiren in keiner Weise von dem Sehvermögen des Anderen und es wäre allen Cataractkranken zu wünschen, dass eine solche successive Entwickelung des Uebels auf beiden Augen stattfände, um dies Verfahren allgemein durchführen zu können. Leider ist dies nicht der Fall, da gerade die Reilungsperiode der Alterscataract, während welcher das Sehvermögen bereits beinahe aufgehoben, aber die Cortikalmassen noch unvollkommen getrübt sind, sich läulig so lange hinzögert, dass die Cataract auf dem zuerst erkrankten Auge erst operabel wird, wenn der Krranke sich nur noch eines geringen Sehvermögens aut dem zuletzt erkrankten Auge erfreut.

Zum Schluss noch einige Betrachtungen über die Coremorphosis auf einem Augr, wenn das andere gesund ist. Man hat auch hiergegen ähnliche Bedenken, wie gegrn die einseitige Cataractoperation erhoben. Obwohl nun dieselbe von mehren Seiten besonders durch Desmarres bekämpft worden sind, so möchte ich meine einschlägigen Ergebnisse öffentlich niederlegen, da i॰h ̈̈her 300 Pupillenbildungen nuter diesen Verhältnissen verrichtet, und nicht versäumt habe, die Rolle des operirten Auges beim gemeinschaftlichen Sehakt zu studiren.

Vor allen Dingen ist es ein durchaus unüberlegter Gegengrund, dass durch die Excentricität der Pupille entweder das operirte Auge in eine schielende Stellung gerathen oder sich vom gemeinschaftlichen Sehakt ausschliessen müsse. Die Excentricität der Pupille wird auf den Lichteinfall ebenso wirken, als wenn wir bei stark erweiterter Pupille deren mittleren Abschnitt verdecken. Hierdurch wird, wie z. B. durch eine centrale Hornhauttrübung, ein Theil des einfallenden Lichtkegels abgeschnitten, aber bei richtigen Accommodationsverhältnissen wird immer das Licht, welches von einem 
in der verlängerten Sehaxe gelegenen Objekte kommt, auf die macula lutea hin sich vereinigen. - Auch hat mir die Erfahrung zur Genüge bewiesen, dass bei allen möglichen Formen von Pupillar - Excentricitäten, seien sie angeboren oder durch Traumen, Krankheiten, Operationen bedingt, ein gemeinschaftlicher Sehakt ohne schielende Stellung vorkommt. Ja wir haben auf die Herstellung eines solchen mit allen daran geknüpften Vortheilen volle Aussicht, wenn die Sehkraft des Auges einigermassen exakt, die Linse erhalten und seitens der Augenmuskeln kein Hinderniss zur Einstellung vorhanden ist. Dies letztere ist namentlich von Wichtigkeit, denn es verringert sich fast bei allen einseitigen Erblindungen die Energie der innern Augenmuskeln zur accommodativen Bewegung.

Die Verhältnisse der Eimrichtung nach ausgeführter Pupillenbildung zeigen übrigens grosse Verschiedenheiten, welche wohl einmal einer gründlichen Analyse bedürften. Es seien hier nur kurz folgende Thatsachen hervorgehoben, damit der obige Satz ,dass ein mit einer excentrischen Pupille versehenes Auge zum Fixiren die Sehaxe auf das Objekt richtet" nicht missgedeutet werde.

1) Es bezieht sich dieser Satz in seiner Strenge nur auf Pupillen, die entweder mässig excentrisch liegen, oder deren einer Abschnitt noch in die mittleren Hornhautpartieen eingreift. Bei ganz excentrischen Pupillen, welche lediglich dem Randtheil der Cornea und des Linsensystems gegenüber liegen, tritt meist ein abweichender Modus des Sehens ein. Es fällt nämlich dann das aus der Verlängerung der Sehaxe stammende Licht so schief auf die Trennungsflächen der brechenden Medien, dass es durch Reflexion zu sehr abgeschwächt wird, um dem Sehakt zu dienen, um so mehr, als es ausserdem höchst unvollkommen auf der Netzhaut vereinigt wird. In diesen Fällen entsteht eine Konkurrenz des centralen Sehens mit dem excentrischen Sehen, welches letztere 
dadurch erreicht wird, lass das Licht bei vorbeischiessender Sehaxe senkrecht zu dem der Pupille entsprechenden Seitentheil der Cornea auftältt. Man sieht alsdann häufig die Patienten zwischen beiden Stellungen wechseln; sie benutzen bald das centrale Sehen mit verhältnissmïissig geringer Lichtstärke, bald das excentrische sehen mit verhältnissmässig grösserer Lichtstïrke, und wählen das eine oder andere zuweilen nach abwechselnden Postulaten des Sehakts. Bei diesen hochgradig schwachsichtigen Augen habe ich ïbrigens einen mit dem gesunden Auge gemeinschaftlichen Sehakt noch nie beobachtet.

2) Erleidet der Satz dadurch Ausnahmen, dass bei adhaerirenden Lenkomen etc. die Krümmung der Hornhaut häulig verändert ist. Es wird hierbei wegen der alienirten Brechungsverhältuisse nicht mehr das in Richtung der Sehaxe einfallende Licht zur macula lutea gelangen, sondem es wird ein in anderer Axe durchgehender Strahl als Axenstrathl tür das centrale. Sehen dienen. Es muss dann bei der Fixation natïlich eine Aberration der sehaxe eintreten.

3 ) Auch Schiefstellung der Linse, auf deren Vorkommen wir schon anderen Orts (siehe Archiv f. O. Bd. I. 2. S. 291) bei synechia anterior autinerksam gemacht haben, kamn in ähnlicher Weise zur Aberration der Sehaxe führen.

4) Handelt es sich überhaupt nicht um geringe, sondern $\mathrm{nm}$ grob nachweisbare Aberrationen, da die nenero Physiologie mit Evidenz erwiesen hat. dass auch miter normalen Verhältnissen nicht die Augenaxe, sondern eine von derselben abweichende, die Hornhaut nach imnen vom vordern Pol treffende, Axe auf das Gesichtsobjekt gerichtet wird. Es dürtte vielleicht diese Thatsache zur Erklärung gewisser Unterschiede in der Sehkraft je nach der Richtung der Pupillarexcentricitäten verwerthet werden. Dass z. B. peripherisch*) nach aussen

*) Bei mässiger Excentricität habo ich solche Untersehiele nieht uachweisen können. 
gelegene Pupillen ceteris paribus ein geringeres Sehvermögen liefern, als nach innen gelegene, ist zwar thatsächlich, aber noch nicht zur Genüge erklärt. Es müsste die Sehschärfe, wemn wir von allen Verhältnissen der Einstellung durch die Muskeln abstrahiren, vollkommen identisch sein, vorausgesetzt, dass die Augenaxe mit der Sehlinie wirklich zusammenfällt, denn es hätten dann alle Theile der Hornhautperipherie dieselbe symmetrische Berechtigung für die dioptrischen Verhältuisse*).

Ist nach einseitiger Pupillenbildung kein gemeinschaftlicher Sehakt vorhanden; so wird uns die Prüfung der etwa künstlich hervorgerufenen Doppelbilder die Ueberzeugung verschaffen, in wiefern ein solcher möglich wäre. So erinnere ich mich zweier Fälle, wo bei einer nach aussen angelegten Pupille und sehr befriedigendem Sehvermögen Doppelbilder zwar nicht spontan, wohl aber angegeben wurden, so wie ich das gesunde Auge mit einem violetten Glase verdeckte. Für die Entfernung standen diese gekreuzten Doppelbilder sehr dicht neben einander, aber bei zunehmender Annäherung trennten sich dieselben mehr und mehr. Die verrichtete Tenotomie des rectus externus, in dem einen Falle total in dem andern partiell, wirkte hier in ähnlicher Weise wie bei Insufficienz der innern Augenmuskeln, und es trat nach derselben ein gemeinschaftlicher Gebrauch der Augen ein. In dem einen Fall, wo die

*) Ich setze hierbei den Fall des centralen Sehens voraus, beim excentrischen Sehen konnten freilich Unterschiede in der Empfünglichkeit der verschiedenen, wenn gleich symmetrisch zur macula lutea gelegenen Netzhauttheile zur Erklärung angerufen werden, aber auch diese sind zur Zeit noch unerwiesen. Ausserdem ist ein Vergleich excentrischer Gesichtseindrücke bei deren Schwäche sehr misslich, und könnte aus solchen Fällen wohl kein Schluss für die Vorzüge, resp. Nachtheile gewisser Pupillenexcentricitäten gezogen werden. 
Tenotomie total vollzogen, war die crste Zeit hindurch eine schwache pathologische Convergenz für entferntere Gegenstände zugegen, mit periodisch auftauchenden gleichnamigen Doppelbildern. Die Diplopie verschwand allmählig, aber man konnte eine Spur von pathologischer Convergenz noch nach einem Vierteljahre nachweisen; dagegen fixirte der Kranke von $6^{\prime \prime}-2^{\prime}$ und hatte innerhalb dieser Grenzen die schärfste Abschätzung der Entfernungen. In dem andern Fall war durch die Tenotomie ein vollständig gemeinschaftlicher Gebrauch der Augen hergestellt,

In sehr vielen Fällen (ich glaube in der Mehrzahl, und konstant bei sehr excentrischen Pupillen) findet nach einseitiger Pupillenbildung kein gemeinschaftlicher Gebrauch statt, und es gelingt uns auf keine Weise, künstliche Doppelbilder hervorzurufen. Es bleiben dann auch nur die generellen Vortheile, nämlich Erweiterung des Gesichtsfeldes und eine allerdings nicht constante Zunahme in der Energie des Sehvermögens.

Ich habe diese letztere zuerst fïr das schielende Auge erwähnt (A. f. O. Bd. I. Abth. 1, pag. 89), und von der grösseren quantitativen Erregung der Netzhïute abhängig gemacht, da sie nicht auf Wahrnehmung des doppelseitigen Lichteindruckes basirt. Seit jener Zeit habe ich eine sehr grosse Anzahl von Kranken und Gesunden hieranf geprüft und Folgendes gefunden:

1) Das Phänomen, dass der zur Unterscheidung nöthige Gesichtswinkel beim doppelseitiget Oeffnen geringer ist, kommt bei vielen Individuen vor, bei sehr vielen nicht und weiss ich über die Gründe dieser Verschiedenheiten zur Zeit nichts zu sagen, da sich in den näheren Verbältnissen bisher nichts Konstantes herausstellte.

2) Wenn das fragliche Phänomen am Gesunden beobachtet wird, so ist es zum grössten Theil durch qua l1titative Erregung der zweiten Netzhaut zu erklären, 
demn es blieb die bessere Sebschärfe in den meisten Fällen, wenn das zweite Auge halb verdeckt und dadurch vom Gesichtsobjekt ausgeschlossen wurde. Ganz ebenso verhält es sich in den uns beschäftigenden Krankheitsfällen, wo kein gemeinschaftlicher Gebrauch der beiden Augen stattfindet.

3) Wesentlich zu unterscheiden von der Zunahme der Sehschärfe ist die grössere Fähigheit Zerstreuungskreise zu überwinden. Diese existirt in der Regel beim doppelseitigen Oeffinen. Sie ist zum Theil eine scheinbare, sofern wegen der grösseren $\mathrm{Pu}$ pillarkontraktion beim doppelseitigen Lichteinfall die Zerstreuungskreise wirklich kleiner werden, zum anderen Theil aber ist sie reell. Ersteres (vermehrte Pupillarkontraktion) hängt bekanntlich nur von der quantitativen Erregung des zweiten Auges, letzteres aber von dem gemeinschaftlichen Sehakt ab. Hiervon überzeugt man sich an solchen Presbyopen, die mit beiden Augen viel näher lesen können, als mit einem. Wird das zweite Auge halb verdeckt, d. h. vom Gesichtsobjekt ausgeschlossen, aber derselbe Contraktionszustand der Pupillen erhalten, so rückt die Grenze des Erkennens meist schon weiter $a b$, obwohl die Zerstreuungskreise gleich gross bleiben; wird nun das zweite Auge ganz verschlossen, so tritt anf Grund der Pupillarerweiterung eine weitere Abrückung jener Grenze ein. A priori könnte freilich vermuthet werden, dass der doppelseitige Sehakt Accommodations-Unterschiede einleite, es war mir aber, wie ich schon früher (1. c.) angab, nicht möglich, solche optometrisch nachzuweisen.

Man hat von der einseitigen Pupillenbildung verschiedene Nachtheile gefürchtet, insonderheit Doppelsehen, Schielen, Störung des Sehaktes durch Blendung. Es handelt sich darum, zu entscheiden, in wiefern diese Nachtheile existiren und den angegebenen Vortheilen gegenüber von Belang sind. Doppelsehen habe ich zuweilen, wiewohl in der unendlichen Minderzahl (nicht mehr als 4 Procent) der Fälle gesehen. Es verschwand 
dies Doppelsehen theils von selbst, indem die Auger. sich zum gemeinschaftlichen Sehakt anschickten, oder indem das Nebenbild des operirten Auges von der Wahrnehmung ausgeschlossen wurde, theils wurde gegen dasselbe durch Tenotomieen eingeschritten. Letztere hatten zuweilen die Bestimmung, einen gemeinschaftlichen Sehakt einzuleiten, zuweilen bezweckten sie lediglich, gekreuzte Doppelbilder in gleichnamige umzuwandeln, als welche dieselben ihren störenden Einfluss (durch Suppression des schwächeren Bildes, hald gänzlich verloren. Schielen - im gröbsten Sinne, d. h. auffallendes Vorbeischiessen der Sehaxe am Gesichtsobjekt - sieht man nicht selten an Augen, welche künstliche Pupillen tragen. In der unendlichen Mehrzahl der Fälle besteht das Schielen aber schon vor der Operation. Es ist ja bekannt, wie häufig Augen mit Leucom und derlei in eine schielende Stellung gerathen. Dass sich das Schielen nicht im Dienste des gemeinschaftlichen Sehaktes entwickelt, habe ich oben hervorgehoben. Wenn wir trotzdem, wiewohl selten, Schielen in Folge der Pupillenbildung beobachten, so hat dies eine ganz andere Entstehungsweise. In einigen Fällen waren es deutlich die gekreuzten Doppelbilder, welche eine zunehmende Divergenz (behufs des grösseren Abstandes der Doppelbilder) hervorriefen. Es entwickelte sich die Ablenkung hier sehr rasch. In den übrigen, spärlichen Fällen trat ohne irgend eine Betheiligung des Auges im gemeinschaftlichen Sehakt allmählig Schielen auf: es waren dies Patienten, bei denen die einseitige Erblindung noch nicht sehr lange bestand, und es ist daher möglich, dass lediglich der fortgesetzte Nichtgebrauch des Auges (beim gemeinschaftlichen Sehakt), wie so häufig, die Ablenkung hervorrief; es ist aber auch möglich, und aus Analogie nicht unwahrscheinlich, dass die verschiedene quantita- 
tive Reizung der peripherischen Netzhauttheile auf dem Wege des Reflexes Augenmuskel-Kontraktionen in entsprechenden Richtungen hervorruft. In Summe sind die Fälle hochgradiger Ablenkungen - und nur von diesen kann die Rede sein, da eine genaue Fixation mit Aufhebung des gemeinschaftlichen Sehaktes erlischt - im Verhältniss zur Zahl der Operirten zu selten, als dass wir in deren Vorkommen eine Gegenanzeige finden künnten, um so mehr, als die etwaige kosmetische Störung allemal durch die Tenotomie zu beseitigen ist. Irgend eine Beeinträchtigung des gemeinschaftlichen Sehakts durch Blend ung wird, wenn wir der Pupille nicht etwa eine übertriebene Grösse geben, niemals beobachtet.

Endlich hat man gegen die Pupillenbildung auf einem Auge den Einwurf gemacht, dass sie den Gesichtsausdruck des Patienten entstelle. Ich will nicht abstreiten, dass eine excentrische Pupille gegenüber einer centralen etwas widernatïrliches, ja selbst etwas entstellendes bietet. Dagegen muss ich den Ausdruck eines mit einer künstlichen Pupille versehenen Auges unendlich günstiger anschlagen, als den eines Auges mit Pupillarverschluss, oder mit leucoma. Dies letztere trägt den Stempel der Blindheit vollkommen deutlich, während es einen lebendigeren Eindruck gewinnt, so wie eine schwarze Pupille, wenn auch excentrisch gelegen, sichtbar wird. Bei Pupillensperre durch feinere Exsudate fällt dies freilich weg, da die Trübung der centralen Pupille für die gröbere physiognomische Beurtheilung nicht eben auffällig war. In solchen Fällen sind es aber wichtigere Indikationen als die kosmetische, welche uns leiten.

Fassen wir dies Alles zusammen, so liegt gegen die Pupillenbildung auf einem Auge kein einziger schlagender Einwand vor, nichts sicherlich, was den bedeu- 
tenden Vorthrilen entgegenzutreten vermöchte. Und selbst wenn wir von allen erörterten Beziehungen abstrahiren wollten, ist es nicht für viele Patienten eine unendliche Beruhigung, zwei Augen statt eines zu besitzen, nicht mehr in der Befürchtung einer völligen Erblindung zu leben? Ist nicht die Einäugigkeit für so manche Arbeiter etwas trübseliges, denen oft temporär das eine Auge unbrauchbar wird, wenn ihnen Staub oder fremde Körper eindringen, oder wenn sie an einer Uphthalmie leiden? Hindert nicht der Vorwurf der Einäugigkeit so Manchen in seiner Laufbahn?

Zu dem kommt, dass die Austührung der Coremorphosis selbst, wenn wir nur vernünftige Grundsätze dabei walten lassen, nicht die geringsten Gefahren bietet, dass der direkte tirfolg immer sicher ist, und das zu erreichende Sehvermögen sich durch genaue Prüfung des Lichtscheins vor der Operation mit einer erl'reulichen Genauigkeit vorausbestimmen lässt. Sind die Verhältnisse in dem kranken Auge ausserordentlich ungünstig, handelt as sich rtwa um Pupillen, die äusserst excentrisch werden, oder doren grösster Theil durch das obere Lid stark vordeckt wird, uder um Pupillen hinter streifig getrübter Hornhaut und derlei, wie wir es wohl auszuführen pflegen, um völlig lirblindeten einen wenn auch kümmerlichen Lichtschein zu verschafien; so würde ich es nicht für allgemein räthlich linden, zu operiren, falls das zweite Auge gresund ist, weil alsdann die Vortheile, welche dem Kranken erwachsen, zu unbedeutend ausfallen. Sind aber dic Verhältnisse auf' dem zweiten Auge einigermassen günstig, so behaupte ich, dass wir durch die Ausführung der Operation dem Kranken wesentliche Dienste leisten. 